\title{
Amphibian Skin Microbiota Response to Variable Housing Conditions and Experimental Treatment across Space and Time
}

\author{
Authors: Vanessa P. Wuerthner, Obed Hernández-Gómez, and Jessica Hua
}

Source: Journal of Herpetology, 53(4) : 324-335

Published By: Society for the Study of Amphibians and Reptiles

URL: https://doi.org/10.1670/18-120

BioOne Complete (complete.BioOne.org) is a full-text database of 200 subscribed and open-access titles in the biological, ecological, and environmental sciences published by nonprofit societies, associations, museums, institutions, and presses.

Your use of this PDF, the BioOne Complete website, and all posted and associated content indicates your acceptance of BioOne's Terms of Use, available at www.bioone.org/terms-o-use.

Usage of BioOne Complete content is strictly limited to personal, educational, and non - commercial use. Commercial inquiries or rights and permissions requests should be directed to the individual publisher as copyright holder. 


\title{
Amphibian Skin Microbiota Response to Variable Housing Conditions and Experimental Treatment across Space and Time
}

\author{
Vanessa P. Wuerthner, ${ }^{1,2,3}$ Obed Hernández-Gómez, ${ }^{1,4}$ and Jessica Hua ${ }^{2}$ \\ ${ }^{2}$ Biological Sciences Department, PO Box 6000, Binghamton University, Binghamton, New York, USA \\ ${ }^{4}$ Department of Environmental Science, Policy and Management, 147 Hilgard Hall, University of California-Berkeley, Berkeley, California, USA
}

\begin{abstract}
Host-associated microbial ecology research is becoming a popular tool in conservation. For amphibians, there is recognition that environmental factors and anthropogenic activities can alter the composition and function of skin microbiotas. Despite growing studies on this topic, the environmental conditions and experimental methods that amphibians are exposed to vary among studies, potentially influencing our ability to develop generalizations. Using Northern Leopard Frogs (Lithobates pipiens), we investigated how the movement of individuals between housing conditions affected the amphibian skin microbial communities. In addition, we evaluated whether variation in experimental venue (e.g., culture pools, mesocosms, or laboratory) and time influenced the effect of a common antibacterial (sulfadimethoxine; SDM) on the skin microbiota. We found that the microbial community diversity decreased when tadpoles were transferred from culture pools to mesocosms and subsequently increased between mesocosms and the laboratory. When comparing the effect of SDM exposure on the skin microbiota of tadpoles across experimental venues (mesocosm and lab), there was no effect of SDM on alpha diversity. However, we noted opposing patterns between the control and SDM-treated individuals within the mesocosm and laboratory groups. In the laboratory, there were differences in the abundances of operational taxonomic units (OTU) while in the mesocosm there were differences in OTU turnover. Finally, we found that SDM treatment on amphibian microbial communities was consistent across time in the laboratory. Because researchers are integrating microbial assessments into our understanding of conservation biology, our results underscore the importance of standard housing conditions and taking into consideration that experimental design may yield variable results.
\end{abstract}

Mounting evidence suggests that the composition and diversity of microbiotas can significantly influence a number of important health-associated functions (Cho and Blaser, 2012) including metabolism (Devaraj et al., 2013), immunity (Thaiss et al., 2016), and energy/nutrient uptake (Krajmalnik-Brown et al., 2012). Given the connection between host health and microbiotas, host-associated microbial ecology research is becoming an increasingly popular tool in the realm of conservation (Redford et al., 2012; Jiménez and Sommer, 2017). For example, in amphibians, which are declining worldwide, cutaneous microbiotas have been heavily investigated because of their involvement in defense against emerging infectious diseases (Woodhams et al., 2014). As a result, laboratory and field-based studies throughout the world have evaluated the effects of a number of biotic and environmental factors on host-associated microbial communities in amphibians (Appendix 1, Table A1). With the rapid increase in amphibian microbiota studies in the past $7 \mathrm{yr}$, it is important to evaluate how experimental approaches may influence the generalizations developed regarding amphibian microbiotas.

Importantly, there is increasing recognition that environmental factors (i.e., captive status or housing conditions) can alter the composition of amphibian skin microbiotas (Becker et al., 2014; Loudon et al., 2014). As a consequence, variation in housing conditions across studies may have significant implications for our understanding of amphibian microbiotas. Yet, across the literature, there is limited consistency in the conditions with the captive history of individuals (e.g., field caught or long-term captive residents) or rearing environment (e.g., seminatural conditions in mesocosms or aseptic conditions in a laboratory), commonly varying across studies (Appendix 1, Table A1). Additionally, it is common for amphibians to be

\footnotetext{
${ }^{1}$ These authors contributed equally to this work.

${ }^{3}$ Corresponding author. E-mail: vwuerth1@binghamton.edu

DOI: $10.1670 / 18-120$
}

raised in one housing environment and moved to another for acclimation prior to experimental manipulation (Jani and Briggs, 2014; Bradley et al., 2015). Variations in housing conditions can introduce a degree of uncertainty in conclusions drawn from the literature and can also make interstudy comparisons difficult. As such, there is a need to evaluate how variable housing conditions influence the microbial ecology of the amphibian skin.

In addition to understanding how housing conditions influence amphibian microbiotas, many studies are focusing on evaluating how experimental manipulation may affect the microbiota. In particular, the effects of anthropogenic activities (i.e., chemical use) on amphibian microbiotas have received much attention (Appendix 1, Table A1). Yet, to date, the evidence for chemical contaminant exposure affecting amphibian skin microbiota has been equivocal (Becker and Harris, 2010; Costa et al., 2016; Hughey et al., 2016). For instance, even when exposed to the same contaminant, the effects on the microbiota can be variable. For example, Hughey et al. (2016) found no effect of fly ash (e.g., coal-combustion byproduct) exposure on the skin microbiota of Spring Peepers (Pseudacris crucifer) in the laboratory, despite finding compositional differences between wild frogs inhabiting clean and contaminated wetlands. Because of the importance of environmental conditions in shaping microbiotas, it is necessary to evaluate how variation in experimental designs (i.e., exposure venue and timing) influences the effects of contaminant exposure on amphibian skin microbiota.

Using Northern Leopard Frogs (Lithobates pipiens), a common amphibian model in the experimental microbiota literature, we asked: 1) How does moving between different amphibian housing conditions influence amphibian skin microbiotas? 2) Are the effects of chemical contaminant exposure on amphibian skin microbiotas consistent across space (i.e., experimental venues)? 3) Are the effects of chemical contaminant exposure 
TABLE 1. Common captive amphibian housing conditions in the literature.

\begin{tabular}{|c|c|c|c|c|c|}
\hline $\begin{array}{l}\text { Housing } \\
\text { condition }\end{array}$ & Purpose & Size & Substrate & $\begin{array}{l}\text { Water } \\
\text { changes }\end{array}$ & Literature \\
\hline $\begin{array}{l}\text { Common garden } \\
\text { culture pools }\end{array}$ & $\begin{array}{l}\text { Typically utilized to raise amphibians before } \\
\text { the start of an experiment. }\end{array}$ & $90-225 \mathrm{~L}$ & None & Yes & $\begin{array}{l}\text { Jani and Briggs, 2014; } \\
\text { Hua et al., 2017; } \\
\text { Wuerthner et al., } \\
2017\end{array}$ \\
\hline Mesocosms & $\begin{array}{l}\text { Seminatural conditions where tadpoles are } \\
\text { members of a larger community. While } \\
\text { mesocosms typically differ across } \\
\text { experiments, they generally contain } \\
\text { communities of zooplankton, periphyton } \\
\text { (i.e., attached algae, cyanobacteria, and other } \\
\text { microbes), phytoplankton (microscopic } \\
\text { autotrophs), and various assemblages of } \\
\text { aquatic vertebrates and invertebrates. }\end{array}$ & $90-1000 \mathrm{~L}$ & $\begin{array}{l}\text { Leaves, soil, } \\
\text { sand, etc. }\end{array}$ & No & $\begin{array}{l}\text { Staley et al., 2010; } \\
\text { Krynak et al., 2017; } \\
\text { Miles et al., 2017; } \\
\text { Wuerthner et al., } \\
2017\end{array}$ \\
\hline $\begin{array}{l}\text { Laboratory } \\
\text { conditions }\end{array}$ & $\begin{array}{l}\text { Highly controlled conditions where multiple } \\
\text { factors (i.e., temperature, photoperiod, } \\
\text { density food availability, etc.) can be held } \\
\text { constant, which allows researchers to isolate } \\
\text { an independent variable of interest. }\end{array}$ & Variable & None & Yes & $\begin{array}{l}\text { Jani and Briggs, 2014, } \\
\text { 2018; Kohl et al., } \\
\text { 2015; Kohl and Yahn, } \\
\text { 2016; Hughey et al., } \\
\text { 2016; Knutie et al., } \\
\text { 2017; Krynak et al., } \\
\text { 2017; Hua et al., } \\
\text { 2017; Bates et al., } \\
\text { 2018 }\end{array}$ \\
\hline
\end{tabular}

on amphibian skin microbiotas consistent across time in laboratory settings?

We predicted that moving individuals from less-realistic common garden housing conditions to more-realistic seminatural outdoor facilities would increase microbial diversity. Additionally, we expected that moving individuals from more-realistic seminatural outdoor facilities to a sterile laboratory setting would decrease microbial diversity. We anticipated that the effect of chemical contaminant exposure would be similar across experimental venues. Finally, we predicted that the effect of contaminant exposure on skin microbiota would be consistent across time in a laboratory setting.

\section{Materials AND Methods}

Amphibian Collection.-On 29 March 2016, we collected 10 partial Northern Leopard Frog egg masses from a pond located near the Purdue Wildlife Area in West Lafayette, Indiana, USA $\left(40.4457^{\circ},-87.0505^{\circ}\right)$. All animals were collected as recently laid egg masses, kept in pond water, and immediately transported to the Ecological Research Facility at Binghamton University, Binghamton, New York, USA, within $24 \mathrm{~h}$ of collection.

Experiment 1: How Does Moving Tadpoles between Different Housing Conditions Influence the Microbiota?-To understand how housing environment influences amphibian microbiotas, we transitioned tadpoles through three commonly used housing conditions: common garden culture pools (i.e., housing environment used to raise amphibians), seminatural mesocosms (i.e., conditions where tadpoles are members of a larger community), and laboratory conditions (i.e., controlled conditions where multiple elements are held constant; Appendix 2, Fig. A1; Table 1).

Common Garden Culture Pools.-First, upon arrival to Binghamton University, we immediately divided the egg masses into two outdoor, 225-L culture pools (Table 1) filled with $\sim 200 \mathrm{~L}$ of well water (5 masses/pool). We covered each culture pool with $70 \%$ shade cloth to prevent colonization by insects and other amphibians. Once embryos hatched and developed into Gosner stage 25 (Gosner, 1960), we fed tadpoles rabbit chow ad libitum. Per the animal care husbandry protocol at Binghamton University, we changed the water in our culture pools every $3 \mathrm{~d}$. To change the water, we first filled a 19-L bucket with well water and netted all individuals from the culture pool into the bucket. We then removed all of the water from the pool and used a scrub brush to remove any attached algae and waste. The pool was then rinsed and refilled with $\sim 200 \mathrm{~L}$ of well water. The bucket was left to float in the culture pool for $30 \mathrm{~min}$ to allow time for water temperatures to acclimate. The tadpoles were then placed into the pool and fed ad libitum.

On 30 June 2016, we haphazardly chose 10 individuals (average mass $=0.245 \pm 0.004 \mathrm{~g}$ ) from each of the two culture pools to swab for skin microbiota. Tadpoles had been in the culture pools for $t=93$ days. Prior to swabbing, new nitrile gloves were donned and each individual was rinsed with 100 $\mathrm{mL}$ sterile water to ensure the sample included predominantly amphibian skin-associated microbes (Culp et al., 2007). Immediately after rinsing, each tadpole was uniformly sampled using a cotton-tipped swab over the entire surface of the body for $30 \mathrm{~s}$ (McKenzie et al., 2012). Individuals were returned to the culture pools immediately after swabbing. Additionally, we swabbed the substrate of each culture pool for $30 \mathrm{~s}$. Each swab was placed in a sterile, $1.5-\mathrm{mL}$ tube and stored at $-80^{\circ} \mathrm{C}$ until DNA extraction.

Semi-Natural Mesocosms.-The next housing environment we used was a seminatural mesocosm (Table 1). To create the mesocosms, on 2 June we filled five, 110-L pools with $95 \mathrm{~L}$ of well water. We then added $5 \mathrm{~g}$ of rabbit chow to serve as an initial nutrient source, $100 \mathrm{~g}$ of oak (Quercus spp.) leaf litter to provide surfaces for natural periphyton growth, and a 1-L aliquot of pond water containing natural periphyton, phytoplankton, and zooplankton assemblages from two local ponds (Nuthatch small: $42.080553^{\circ},-75.985425^{\circ}$ and Nuthatch large: $42.080167^{\circ}$, $-75.985595^{\circ}$ ) to each pool. We covered each mesocosm with a $70 \%$ shade cloth lid to prevent colonization by insects and other amphibians. We allowed the pools to acclimate for $4 \mathrm{wk}$ before the addition of tadpoles. On 1 July, to mimic natural amphibian densities (Werner and Glennemeier, 1999; Parris et al., 2006), we 
randomly selected and moved 25 individual Leopard Frog tadpoles from the culture pools to each mesocosm. On 11 July, we haphazardly chose five individuals from each of the five mesocosms to swab for skin microbiota using the same methods described above. Additionally, we swabbed the substrate of each mesocosm.

Laboratory Conditions.-On 11 July, we haphazardly selected and transferred 10 tadpoles from the seminatural mesocosms into a temperature- $\left(20^{\circ} \mathrm{C}\right)$ and light-controlled $(12: 12$ light : dark cycle) laboratory setting (Table 1 ). We individually placed all tadpoles into separate plastic 2-L tubs filled with $1 \mathrm{~L}$ of filtered, ultraviolet (UV)-irradiated aged well water. Tadpoles were fed a $1: 1$ ground TetraMin mixed with water diet ad libitum every $2 \mathrm{~d}$ and we conducted water changes every $5 \mathrm{~d}$. On 21 July and 31 July, we swabbed the 10 individuals to assess their skin microbiota (McKenzie et al., 2012).

Experiment 2: Is the Effect of Contaminant Exposure on the Microbiota Consistent across Space and Time?-To understand whether experimental venues modify the effect of contaminant exposure on amphibian microbiotas, we chose to work with sulfadimethoxine (SDM; 4-amino-N-2,6-dimethoxy-4-pyrimidin$\mathrm{yl}$ ). SDM is a broad-spectrum sulfonamide antimicrobial commonly used to treat and prevent infections in agricultural livestock (i.e., chickens, cattle, pigs). SDM inhibits the synthesis of folic acid by fungal and bacterial microorganisms (Applegate, 1983). SDM is excreted in cattle urine and has been detected as a metabolite in excrement (Bourne et al., 1981). Manure is regularly applied to fields; therefore, SDM can run off into aquatic systems (Kreuzig et al., 2005; Hamscher et al., 2006). Concentrations up to $36 \mu \mathrm{g} / \mathrm{L}$ and $703 \mu \mathrm{g} / \mathrm{L}$ of SDM have been detected in ponds and agricultural runoff, respectively, though lower concentrations ranging from 0.88 to $2 \mu \mathrm{g} / \mathrm{L}$ are more common (Kreuzig et al., 2005; Hamscher et al., 2006). Notably, we focused on SDM because we previously showed that SDM can alter the microbial beta diversity of amphibian skin microbiota (Hernández-Gómez et al., 2019).

On 2 June, we created five seminatural aquatic communities that contained a concentration of $1 \mu \mathrm{g} / \mathrm{L}$ SDM. To create the 1 $\mu \mathrm{g} / \mathrm{L}$ of SDM solution in the mesocosms, we first mixed a 0.63 $\mu \mathrm{g} / \mathrm{L}$ SDM stock solution by dissolving $4.989 \mathrm{mg}$ of SDM (Durvet sulfadimethoxine soluble powder, Anada 200-376) in 7 $\mathrm{L}$ of well water. Next, to achieve a concentration of $1 \mu \mathrm{g} / \mathrm{L}$ of $\mathrm{SDM}$ in each of the mesocosms, we added $150 \mathrm{~mL}$ of the 0.63 $\mu \mathrm{g} / \mathrm{L}$ SDM stock solution to each pool. This portion of the experiment was concurrently conducted using identical mesocosm conditions to those described in Experiment 1. This design allows for the direct comparison of the skin microbiota of tadpoles not exposed vs. exposed to SDM. Using the same dosing protocol, we again added SDM to each mesocosm on 5 July and 9 July. On 11 July, we swabbed the substrate of each mesocosm and haphazardly chose five individuals from each of the mesocosms to swab.

Next, to investigate whether the SDM treatment on the amphibian microbiota was consistent across different experimental venues, on 11 July we transferred 10 tadpoles from the 1 $\mu \mathrm{g} / \mathrm{L}$ of SDM seminatural mesocosms into a laboratory setting. Upon arriving in the lab, we allowed all tadpoles to acclimate in $1 \mathrm{~L}$ of UV-filtered SDM-free well water for $48 \mathrm{~h}$ (Bradley et al., 2015). Then we replaced the filtered well water with $1 \mu \mathrm{g} / \mathrm{L}$ of SDM solution. Subsequent water changes were conducted every $5 \mathrm{~d}$ at which point we renewed the $1 \mu \mathrm{g} / \mathrm{L}$ SDM treatments. All experimental units (mesocosms and tubs) were dosed using a newly mixed SDM stock solution. Additionally, to ensure that the commercial-grade antibiotic contained accurate concentrations of the active ingredient, SDM, we sent the nominal 0.63 $\mu \mathrm{g} / \mathrm{L}$ SDM stock solution to the University of Nebraska Spectroscopy and Biophysics Core for testing. The actual concentration of our stock solution was $0.63 \pm 0.14 \mu \mathrm{g} / \mathrm{L}$ (average \pm standard error), confirming that nominal and actual concentrations of the commercial grade product were consistent. This part of the experiment was simultaneously conducted using the identical laboratory conditions as those described in Experiment 1, allowing for the direct comparison of the skin microbiota of tadpoles not exposed vs. exposed to SDM. On 21 July and 31 July, we swabbed each of the 10 individuals to assess whether the amphibian skin microbiota was consistent across time (McKenzie et al., 2012).

Microbiota Laboratory Methods.-We isolated DNA from skin swabs using the PowerSoil DNA Isolation Kit (MoBio Laboratories Inc., Carlsbad, CA) following the protocol described in Hernández-Gómez et al. (2017a). In brief, we implemented two sequential PCRs to prepare our 16S rRNA V2 region amplicon sequencing library using the primer pair 27F/338R (Fierer et al., 2008) and following the methodology described in HernándezGómez et al. (2017b). We shipped the barcoded sample pool on dry ice overnight to the Cornell University Biotechnology Resource Center (Ithaca, NY). We sequenced the sample pool on a MiSeq machine (Illumina, Inc. San Diego, CA) using the reagent kit V2 to produce 250 base pair (bp) paired-end reads. The microbiota sequencing library preparation for this study was produced in combination with a previous study (HernándezGómez et al. 2019).

Amplicon Sequence Analysis.-We processed raw sequencing reads using Trimmomatic (Bolger et al., 2014) to remove adapter sequences, bases below threshold quality of phred-20 from both ends of reads, and any resulting reads under $30 \mathrm{bp}$. We paired reads that passed initial quality control using PANDAseq (Masella et al., 2012) and only retained reads that paired successfully. We uploaded the raw sequencing data to the National Center for Biotechnology Information (NCBI, Bethesda, MD; https://www.ncbi.nlm.nih.gov/) Sequence Read Archive under the project accession number PRJNA429454.

We implemented the Quantitative Insights Into Microbial Ecology version 1.9.0 (QIIME) pipeline (Caporaso et al., 2010) to filter erroneous reads, cluster reads into operational taxonomic units (OTU) at the standard 97\% identity using the openreference protocol, and generate abundance-based OTU tables. To avoid including any OTUs generated by sequencer error, such as base miscalls or chimeras, we performed additional quality filtration on the OTU table by removing OTUs that were represented by fewer than $0.005 \%$ of the total read count (Bokulich et al., 2013). We also removed any OTUs that matched in taxonomy to chloroplast or mitochondria. To standardize sequencing depth across all samples, we rarefied the OTU table to 11,305 sequences per sample.

Statistical Analysis. - We transferred the rarefied OTU table and Newick phylogenetic tree to $\mathrm{R}$ (version 3.2.0, $\mathrm{R}$ Core Team, Vienna, Austria, available from https://www.R-project.org). We calculated the proportion of shared OTUs in tadpole microbiotas between culture pool and mesocosm and the corresponding substrate samples. To evaluate alpha diversity (i.e., OTU richness or diversity within each sample), we calculated community richness (observed OTUs) and diversity (Shannon diversity and Faith's phylogenetic diversity) using the packages vegan (Oksanen et al., 2013) and Picante (Kembel et al., 2010). In addition, we implemented the packages GuniFrac (Chen et al., 

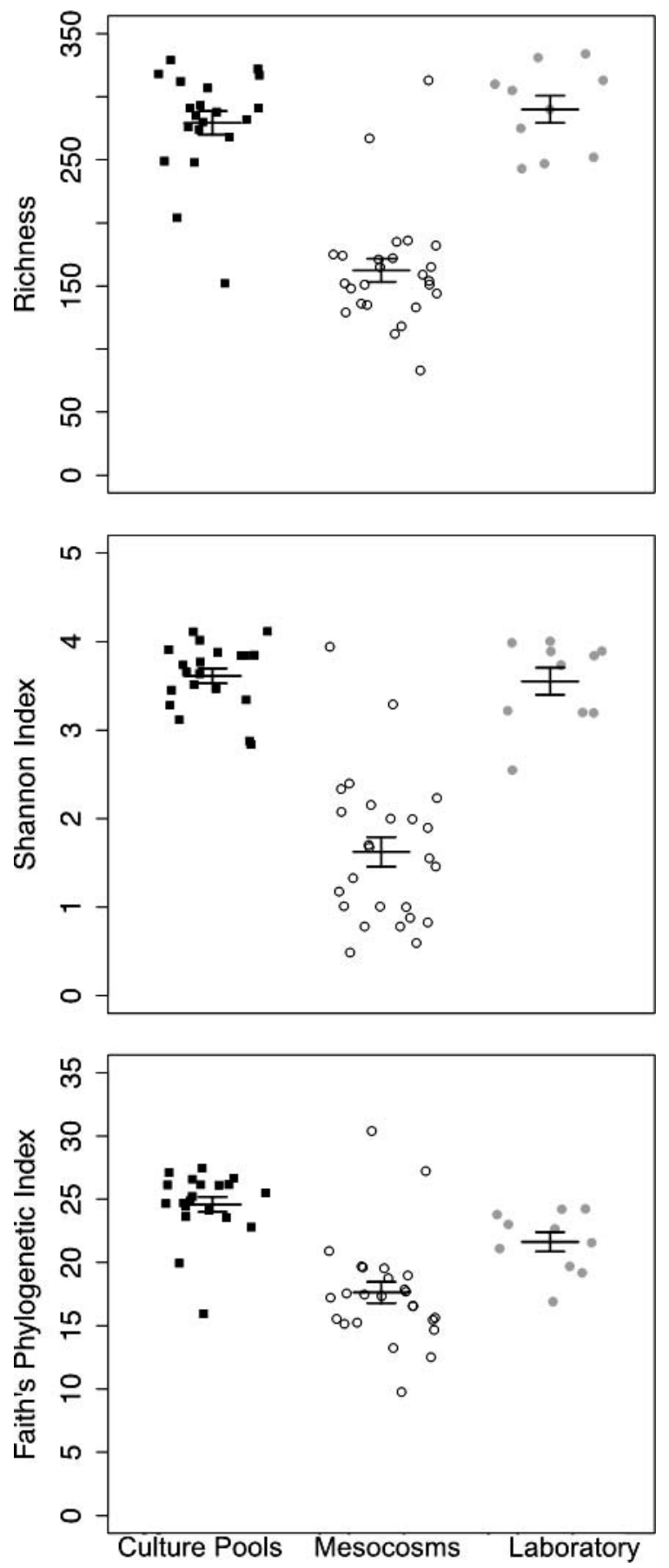

FIG. 1. Alpha diversity values measured from the skin of Northern Leopard Frog tadpoles in three different environments (culture pools, mesocosms, and laboratory). Long horizontal lines representing the mean and standard error lines are presented.

2012) and vegan to calculate three beta diversity metrics (i.e., community composition differences between samples): unweighted UniFrac, weighted UniFrac, and Bray-Curtis distances. For a detailed description of each statistical analysis, refer to Appendix 3.

\section{Results}

From 120 skin and substrate swab samples combined, 16S rRNA V2 amplicon sequencing resulted in 2,109,481 reads. After filtering out reads by base pair length, we processed the remaining reads through QIIME (http://qiime.org/) using the open reference clustering method to return 852 OTUs for all skin samples after OTU abundance filtration and rarefaction.

Experiment 1: Influence of Moving Tadpoles between Housing Conditions on Skin Microbiota.-The percentage of shared OTUs between tadpole skin and unit substrate was not significantly associated with housing condition (culture pools or mesocosms; $\mathrm{LRT}_{4}=0.13, P=0.715$ ); yet seminatural mesocosm substrate possessed richer communities than did the culture pool substrate (culture pool $=213 \pm 14$; mesocosms $=298.2 \pm 20.4$ ). We found a significant difference in alpha diversity between housing conditions with individuals in mesocosms possessing less rich/ diverse communities than did individuals from culture pools and laboratory conditions (richness: $\mathrm{LRT}_{5}=12.17, P=0.002$; Shannon index: $\mathrm{LRT}_{5}=12.06, P=0.002$; Faith's phylogenetic diversity: $\mathrm{LRT}_{5}=9.18, P=0.010$; Fig. 1 ). These patterns likely reflect changes observed in the relative abundance of dominant OTUs, as one OTU dominated the community in the mesocosms compared to the laboratory/culture pool samples (Appendix 2, Fig. A2-A3). We also noted differences in beta diversity among housing conditions (weighted UniFrac: $F_{2,54}=20.92, r=0.45, P=$ 0.001; unweighted Unifrac: $F_{2,54}=16.62, r=0.39, P=0.001$; Bray-Curtis: $\left.F_{2,54}=29.04, r=0.53, P=0.001\right)$. Interestingly, the majority of the differentiation visualized through principal coordinates analysis (PCoA) denoted differences between culture pools and mesocosms, with laboratory samples intermediate between the two (Fig. 2).

Experiment 2: Effect of SDM on Microbiotas across Space and Time.-SDM did not have an effect on the alpha diversity of amphibian microbiotas in either mesocosms or laboratory conditions (richness: $\mathrm{LRT}_{5}=0.04, P=0.834$; Shannon index: $\mathrm{LRT}_{5}=2 \times 10^{-3}, P=0.962$; Faith's phylogenetic diversity: $\left.\mathrm{LRT}_{5}=5.01, P=0.171\right)$. When evaluating the effect of housing condition on differences in beta diversity between control and SDM-exposed samples, we found significant differences between mesocosm and laboratory groups for all three distances tested (unweighted UniFrac: $\mathrm{LRT}_{4}=11.93, P<$ 0.001; weighted UniFrac: $\mathrm{LRT}_{4}=2.32, P=0.128$; Bray-Curtis: $\left.\mathrm{LRT}_{4}=11.10, P<0.001\right)$. However, there were contradicting patterns between presence/absence- and abundance-based dissimilarity metrics. We observed larger weighted UniFrac and Bray-Curtis (i.e., abundance-based) differences between control and SDM-exposed individuals in the laboratory (Fig. 3). Conversely, there were larger unweighted UniFrac (i.e., presence/absence-based) differences between control and SDM-exposed individuals in the mesocosm environment (Fig. 3 ). The indicator species analysis performed on the mesocosm and laboratory samples resulted in nearly distinct lists of treatment-associated OTUs. However, OTUs assigned to the class Betaproteobacteria and family Sinobacteraceae were abundant in both antibiotic groups whereas an OTU assigned to the genus Acinetobacter was associated with both control groups.

In the laboratory setting, alpha diversity metrics were comparable between time points and treatment groups, with no significant interactions observed (Table 2). However, Adonis tests revealed significant grouping of communities based on treatment (control vs. SDM) at each time point. Average beta diversity differences between control and SDM samples did not differ between time points for all metrics tested (weighted UniFrac: $F_{1,17}=0.82, P=0.377$; unweighted UniFrac: $F_{1,17}=$ 0.92, $P=0.351$; Bray-Curtis: $F_{1,17}=0.05, P=0.946$ ). 

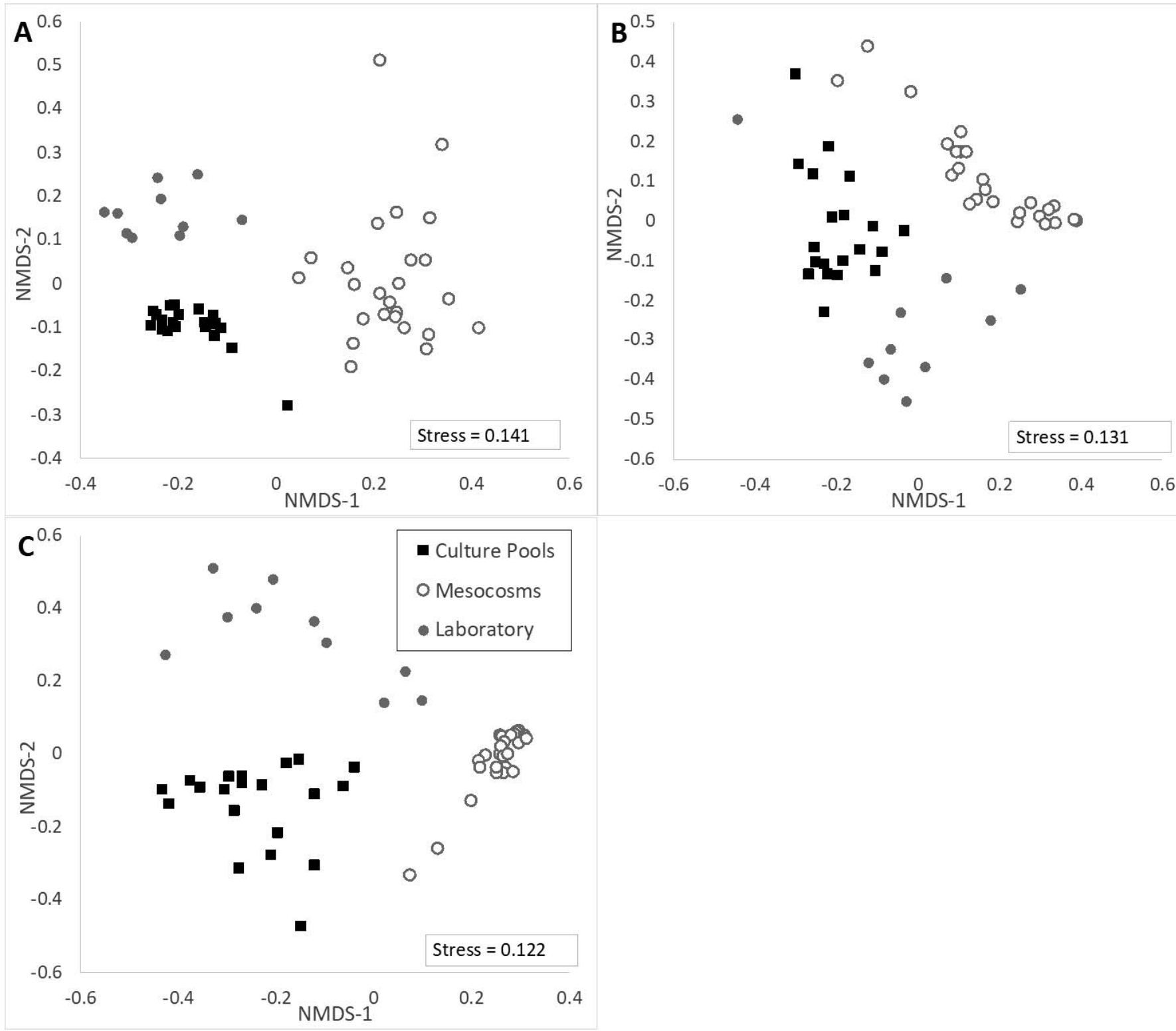

FIG. 2. Nonmetric multidimensional scaling (NMDS) plot of Northern Leopard Frogs raised in three separate environments. Analyses depict coordinates derived using (A) unweighted UniFrac, (B) weighted UniFrac, and (C) Bray-Curtis distance matrices.

\section{DisCUSSION}

Influence of Moving Tadpoles between Housing Conditions on the Skin Microbiota.-We observed a loss of community diversity when tadpoles were transferred from culture pools to mesocosms and a subsequent gain of diversity between mesocosms and laboratory conditions. This is intriguing given that we anticipated a loss of diversity in the aseptic laboratory conditions. One possible explanation for the observed patterns in alpha diversity is the differential response between abundant and rare skin microbes to changes in the environment (Loudon et al., 2014). In fact, in the seminatural mesocosms, there was an increase in the abundance of a ubiquitous OTU identified to the family Commamonadaceae. In this case, either the expansion of Commamonadaceae was associated with a loss of rare species in the mesocosms or the proliferation of Commamonadaceae increased the skew in OTU frequencies, impairing our ability to detect rare species via our methodology. A similar pattern has been observed in Red-Backed Salamanders (Plethodon cinereus), where a negative relationship was observed between the relative abundance of core OTUs and alpha diversity in salamanders kept in sterile conditions (Loudon et al., 2014).

Multiple studies in amphibians and other systems have documented the effects of environmental changes on the composition of host-associated microbiotas (Becker et al., 2014; Loudon et al., 2014). Indeed, we demonstrate that movement between housing conditions also contributed to changes in beta diversity. We noted the biggest shift in both presence/absence and abundance-based beta diversity metrics between the culture pools and the mesocosms, with intermediate metrics from laboratory conditions. Similarities in beta diversity between culture pools and laboratory conditions likely results from resemblances in the environment between the two housing conditions (e.g., absence of substrate and repeated water changes with unfiltered or filtered/UV-radiated well water), which can benefit certain members of the amphibian skin microbiota (Becker et al., 2014). 


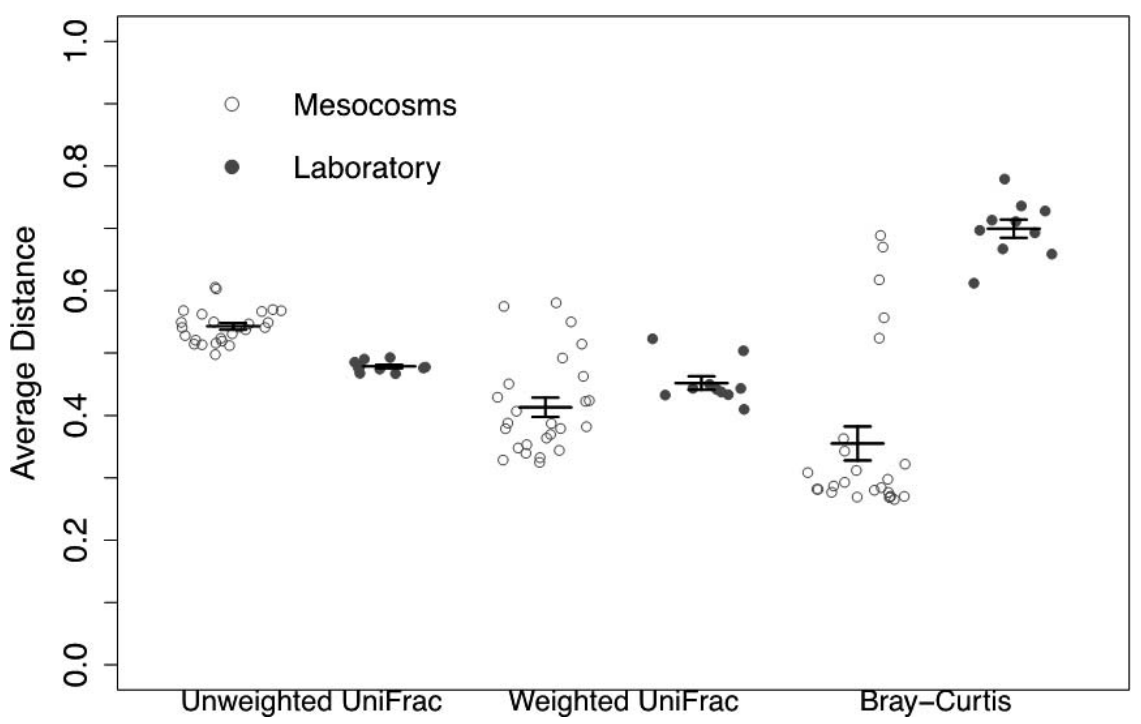

FIG. 3. Average pairwise distances between control and SDM exposed individuals in mesocosm and laboratory environments. Average distances are presented for three different distance metrics; unweighted UniFrac (presence/absence-based), weighted UniFrac (abundance-based), and BrayCurtis (abundance-based). Long horizontal lines representing the mean and standard error lines are presented.

Collectively, our results suggest that more-controlled environments (culture pools and lab) selected for more-diverse and evenly distributed communities whereas seminatural environments (mesocosms) selected for less-diverse and unevenly distributed communities. However, we focused on only three housing conditions using amphibians collected from the wild as eggs. Additionally, we acknowledge that water quality measurements (i.e., ammonia and nitrite) could vary between our treatments and ultimately affect microbial community diversity. While our results suggest that transitioning between housing conditions leads to variation in the diversity of starting microbiota, an important next step is to understand whether patterns in microbiota alpha and beta diversity are generalizable across other variations of controlled and seminatural conditions and across amphibians collected at different life stages. Developing microbiota-specific standardized housing conditions or identifying husbandry practices for microbiota research will become increasingly important as microbiota assessments become more common in conservation biology.

Effect of SDM Treatment on Microbiotas across Space.-When comparing the effect of SDM exposure on the skin microbiota of tadpoles between the mesocosms and laboratory conditions, there was no effect of SDM on alpha diversity. However, we noted contradicting effects of SDM exposure on beta diversity. In the laboratory, there were significant differences in the abun- dances of OTUs between control and SDM-treated individuals. However, in the mesocosm we did not observe differences in OTU abundances but instead observed significant differences in OTU turnover (presence/absence) between control and treatment groups. The effect of SDM on the detection of OTUs in mesocosms was unexpected because of the bacteriostatic function of SDM (Applegate, 1983). Because SDM is predicted to impact the growth of certain bacteria, its application is predicted to result in a shift in abundance of OTUs rather than in complete turnover (Gutiérrez et al., 2010). However, the uneven abundance distribution of OTUs in amphibians from mesocosms (Experiment 1) likely compromised our ability to detect changes in relative abundance even if SDM induced changes in the abundance of OTUs.

Overall, our results suggest that the initial distribution of bacterial species frequencies (uneven vs. even) can influence conclusions drawn regarding how contaminant exposure affects amphibian skin microbiotas. In the case of SDM, even OTU distributions (i.e., achieved via laboratory conditions) may bias results toward shifts in abundance. In contrast, uneven OTU distributions (i.e., achieved via mesocosm conditions) may bias results toward shifts in OTU presence/absence (OTU turnover). Therefore, in order to develop more-accurate generalizations about the effect of contaminant exposure on amphibian microbiotas, future studies might consider characterizing the

TABLE 2. Alpha diversity linear model and beta diversity multivariate statistics testing the effect of SDM (alpha and beta) and time (alpha only) on the skin microbiota of Northern Leopard Frogs. For Beta diversity comparisons, collection times A and B correspond with 21 and 31 July 2016 , respectively.

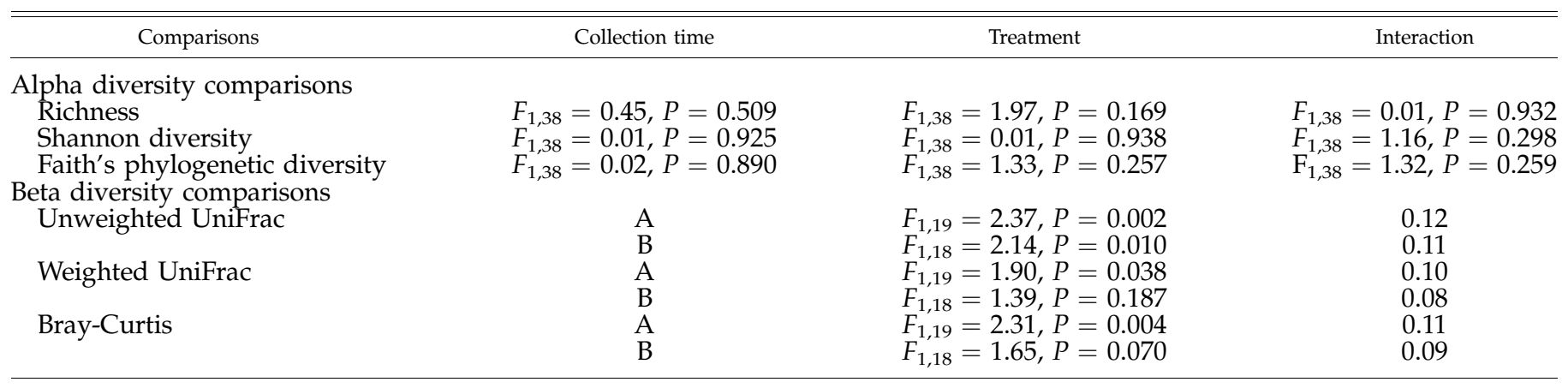


frequency of bacteria on the amphibian skin prior to experimentation.

Effect of SDM on Microbiotas across Time.-We found that in laboratory conditions, the effect of SDM on amphibian microbial communities was consistent across time (days 10 and 20). Recent laboratory studies have demonstrated similar patterns, with experimental manipulation both inducing and maintaining a change in amphibian skin microbiota diversity (Loudon et al., 2014; Warne et al., 2017). Our results suggest that even with frequent water changes, controlled laboratory conditions are ideal for maintaining a stable environment for microbial communities. This stability may have resulted from the limited opportunity for symbiont reseeding, especially given that we maintained constant tadpole conditions in the laboratory between days 10 and 24 (e.g., equal concentrations of SDM, a common housing environment, all tadpoles fed the same diet). Aseptic captive environments tend to lack environmental microbial reservoirs, presence of parents/conspecifics that serve as vertical/horizontal microbial reservoirs, and important dietary prebiotics that may seed or maintain the tadpole skin communities (Antwis et al., 2014; Becker et al., 2014). In nature, reseeding of microbes may be an important mechanism to allow amphibian microbiotas to recover following contaminant exposure. Thus, while laboratory settings may provide more-stable conditions for understanding how microbiotas respond to contaminant exposure, studies that incorporate a longitudinal design in seminatural conditions are necessary to evaluate how environmental reservoirs contribute to the recovery of skin microbiota following perturbation.

Conclusions.-Understanding how microbiotas regulate basic biological processes has received tremendous attention. With the methods for assessing microbiotas becoming more efficient and affordable, an increasing number of researchers are integrating microbial assessments into our understanding of conservation biology. However, this expansion in research has resulted in a wide diversity of experimental techniques. Given the novelty of microbiota-based approaches in amphibian experimental studies, this is a good time to evaluate how these different experimental techniques may influence results. We found that housing condition is important in shaping initial skin microbial communities, underscoring the importance of standard amphibian housing for development of microbiota-specific husbandry best practices.

Additionally, experimental treatments had contradicting effects on microbial composition in mesocosms vs. laboratory conditions, indicating the potential for equivocal results if experimental venue is not taken into consideration. Finally, in laboratory conditions, experimental treatments created stable microbial communities over time. However, interpretations are limited given the lack of reseeding and the potential for microbiotas to recover from perturbation. Therefore, conclusions generated in laboratory-based studies may not represent what occurs in natural environments. Ultimately, our study emphasizes the importance of considering the effects of methodological approaches on skin-associated microbial ecology from the collection of animals through experimental manipulation.

Acknowledgments.-We thank the Purdue University Department of Forestry and Natural Resources Genetics Laboratory for allowing us to perform the microbiota laboratory work in their facility. We also thank the staff of the Cornell University Biotechnology Resource Center for their assistance in sequenc- ing. Finally, we thank N. Buss for collecting the Leopard Frog egg masses. All animals were handled in accordance to the Binghamton University IACUC protocol 757-16. Funding for this research was provided by the NSF DEB 1655190 to JH and the NSF PRFB 1708926 to OH-G.

\section{Literature Cited}

Antwis, R. E., R. L. Haworth, D. J. P. Engelmoer, V. Ogilvy, A. L. Fidgett, AND R. F. PreziosI. 2014. Ex situ diet influences the bacterial community associated with the skin of red-eyed tree frogs (Agalychnis callidryas). PLoS One 9:e85563.

Applegate, J. 1983. Clinical pharmacology of sulfonamides. Modern Veterinary Practice 667-669.

Bates, K. A., F. C. Clare, S. O'Hanlon, J. Bosch, L. Brookes, K. Hopkins, E. J. McLaughlin, O. Daniel, T. W. J. Garner, M. C. Fisher, et al. 2018. Amphibian chytridiomycosis outbreak dynamics are linked with host skin bacterial community structure. Nature Communications 9: 693.

BECKER, M. H., AND R. N. HARRIs. 2010. Cutaneous bacteria of the redback salamander prevent morbidity associated with a lethal disease. PLoS One 5:e10957.

Becker, M. H., C. L. Richards-Zawacki, B. Gratwicke, and L. K. Belden. 2014. The effect of captivity on the cutaneous bacterial community of the critically endangered Panamanian golden frog (Atelopus zeteki). Biological Conservation 176:199-206.

Bokulich, N. A., S. Subramanian, J. J. Faith, D. Gevers, J. I. Gordon, R. KNIGHT, D. A. Mills, AND J. G. CAPORASO. 2013. Quality-filtering vastly improves diversity estimates from Illumina amplicon sequencing. Nature Methods 10:57-59.

Bolger, A. M., M. Lohse, AND B. Usadel. 2014. Trimmomatic: a flexible trimmer for Illumina sequence data. Bioinformatics 30:2114-2120.

Bourne, D. W. A., M. Bialer, L. W. DitTert, M. Hayashi, G. Rudawsky, G. D. Koritz, AND R. F. Bevill. 1981. Disposition of sulfadimethoxine in cattle: inclusion of protein binding factors in a pharmacokinetic model. Journal of Pharmaceutical Sciences 70:1068-1072.

Bradley, P. W., S. S. Gervasi, J. Hua, R. D. Cothran, R. A. Relyea, D. H. Olson, and A. R. Blaustein. 2015. Differences in sensitivity to the fungal pathogen Batrachochytrium dendrobatidis among amphibian populations. Conservation Biology 29:1347-1356.

Caporaso, J. G., J. Kuczynski, J. Stombaugh, K. Bittinger, F. D. Bushman, E. K. Costello, N. Fierer, A. Gonzalez Peña, J. K. Goodrich, J. I. GORDON, ET AL. 2010. QIIME allows analysis of high-throughput community sequencing data. Nature Methods 7:335-336.

Chen, J., K. Bittinger, E. S. Charlson, C. Hoffmann, J. Lewis, G. D. Wu, R. G. Collman, F. D. Bushman, AND H. Li. 2012. Associating microbiome composition with environmental covariates using generalized UniFrac distances. Bioinformatics 28:2106-2113.

CHO, I., AND M. J. BLASER. 2012. The human microbiome: at the interface of health and disease. Nature Reviews Genetics 13:260-270.

Costa, S., I. Lopes, D. N. Proença, R. Ribeiro, and P. V. Morais. 2016. Diversity of cutaneous microbiome of Pelophylax perezi populations inhabiting different environments. Science of The Total Environment 572:995-1004.

Culp, C. E., J. O. FalKinham, AND L. K. Belden. 2007. Identification of the natural bacterial microflora on the skin of eastern newts, bullfrog tadpoles and redback salamanders. Herpetologica 63:66-71.

Devaraj, S., P. Hemarajata, and J. Versalovic. 2013. The human gut microbiome and body metabolism: implications for obesity and diabetes. Clinical Chemistry 59:617-628.

Fierer, N., M. Hamady, C. L. Lauber, and R. Knight. 2008. The influence of sex, handedness, and washing on the diversity of hand surface bacteria. Proceedings of the National Academy of Science USA 105: 17994-17999.

GosNER, K. L. 1960. A simplified table for staging anuran embryos and larvae with notes on identification. Herpetologica, 16:183-190.

Gutiérrez, I. R., N. Watanabe, T. Harter, B. Glaser, and M. RadKe. 2010. Effect of sulfonamide antibiotics on microbial diversity and activity in a Californian Mollic Haploxeralf. Journal of Soils and Sediments 10:537-544.

Hamscher, G., B. Priess, ANd H. NAu. 2006. A survey of the occurrence of various sulfonamides and tetracyclines in water and sediment samples originating from aquaculture systems in Northern Germany in summer 2005. Archiv Fur Lebensmittelhygiene 57:97-101. 
Hernández-Gómez, O., J. T. Hoverman, and R. N. Williams. 2017a. Cutaneous microbial community variation across populations of eastern hellbenders (Cryptobranchus alleganiensis alleganiensis). Frontiers in Microbiology 8:1379.

Hernández-Gómez, O., S. J. A. Kimble, J. T. Briggler, and R. N. Williams. $2017 \mathrm{~b}$. Characterization of the cutaneous bacterial communities of two giant salamander subspecies. Microbial Ecology 73:445-454.

HernánDEZ-Gómez, O., V. P. Wuerthner, AND J. Hua. 2019. Amphibian host and skin microbiota response to a common agricultural antimicrobial and internal parasite. Microbial Ecology https://doi. org.proxy.binghamton.edu/10.1007/ s00248-019-01351-5.

Hua, J., V. P. Wuerthner, D. K. Jones, B. Mattes, R. D. Cothran, R. A. Relyea, and J. T. Hoverman. 2017. Evolved pesticide tolerance influences susceptibility to parasites in amphibians. Evolutionary Applications 10:802-812.

HugheY, M. C., J. B. Walke, M. H. Becker, T. P. Umile, E. A. Burzynski, K. P. C. Minbiole, A. A. Iannetta, C. N. Santiago, W. A. Hopkins, and L. K. BELDEN. 2016. Short-term exposure to coal combustion waste has little impact on the skin microbiome of adult spring peepers (Pseudacris crucifer). Applied and Environmental Microbiology 82: 3493-3502.

JANI, A. J., AND C. J. BRIGGS. 2014. The pathogen Batrachochytrium dendrobatidis disturbs the frog skin microbiome during a natural epidemic and experimental infection. Proceedings of the National Academy of Sciences 111:E5049-E5058.

- 2018. Host and aquatic environment shape the amphibian skin microbiome but effects on downstream resistance to the pathogen Batrachochytrium dendrobatidis are variable. Frontiers in Microbiology.

JiMÉNEZ, R. R., AND S. SOMMER. 2017. The amphibian microbiome: natural range of variation, pathogenic dysbiosis, and role in conservation. Biodiversity and Conservation 26:763-786.

Kembel, S. W., P. D. Cowan, M. R. Helmus, W. K. Cornwell, H. Morlon, D. D. Ackerly, S. P. Blomberg, and C. O. WebB. 2010. Picante: R tools for integrating phylogenies and ecology. Bioinformatics 26:14631464.

KNUTIE, S. A., C. L. Wilkinson, K. D. KoHL, And J. R. RoHr. 2017. Early-life disruption of amphibian microbiota decreases later-life resistance to parasites. Nature Communications 8:86.

Kohl, K. D., T. L. Cary, W. H. Karasov, and M. D. Dearing. 2015. Larval exposure to polychlorinated biphenyl 126 (PCB-126) causes persistent alteration of the amphibian gut microbiota. Environmental Toxicology and Chemistry 34:1113-1118.

KOHL, K. D., AND J. YAHN. 2016. Effects of environmental temperature on the gut microbial communities of tadpoles. Environmental Microbiology 18:1561-1565.

Krajmalnik-Brown, R., Z.-E. Ilhan, D.-W. Kang, and J. K. DiBaise. 2012. Effects of gut microbes on nutrient absorption and energy regulation. Nutrition in Clinical Practice 27:201-214.

Kreuzig, R., S. Höltge, J. Brunotte, N. Berenzen, J. Wogram, and R. Schulz. 2005. Test-plot studies on runoff of sulfonamides from manured soils after sprinkler irrigation. Environmental Toxicology and Chemistry 24:777-781.
KRYNAK, K. L., D. J. BURKE, AND M. F. BenARD. 2017. Rodeo ${ }^{\mathrm{TM}}$ herbicide negatively affects Blanchard's cricket frogs (Acris blanchardi) survival and alters the skin-associated bacterial community. Journal of Herpetology 51:402-410.

Loudon, A. H., D. C. Woodhams, L. W. Parfrey, H. Archer, R. Knight, V. McKenZIE, and R. N. HaRrIs. 2014. Microbial community dynamics and effect of environmental microbial reservoirs on red-backed salamanders (Plethodon cinereus). The ISME Journal 8:830-840.

Masella, A. P., A. K. Bartram, J. M. TruszKowski, D. G. Brown, and J. D. NEUfELD. 2012. PANDAseq: paired-end assembler for Illumina sequences. BMC Bioinformatics 13:31.

McKenzie, V. J., R. M. Bowers, N. Fierer, R. Knight, and C. L. Lauber. 2012. Co-habiting amphibian species harbor unique skin bacterial communities in wild populations. The ISME Journal 6:588-596.

Miles, J. C., J. Hua, M. S. Sepulveda, C. H. Krupke, and J. T. Hoverman. 2017. Effects of clothianidin on aquatic communities: evaluating the impacts of lethal and sublethal exposure to neonicotinoids. PLoS One 12:e0174171.

Oksanen, J., F. G. Blanchet, M. Friendly, R. Kindt, P. Leggendre, D. McGlinn, P. R. Minchin, R. B. O'Hara, G. L. Simpson, P. Solymos, et AL. 2013. Package "vegan": Community Ecology Package version 2.9. http:/ /CRAN.Rproject.org/package=vegan.

PARRIS, M. J., E. REESE, AND A. STORFER. 2006. Antipredator behavior of chytridiomycosis-infected northern leopard frog (Rana pipiens) tadpoles. Canadian Journal of Zoology 84:58-65.

Redford, K. H., J. A. Segre, N. Salafsky, C. M. del Rio, and D. McAloose. 2012. Conservation and the microbiome. Conservation Biology 26: 195-197.

Staley, Z. R., J. R. Rohr, and V. J. Harwood. 2010. The effect of agrochemicals on indicator bacteria densities in outdoor mesocosms. Environmental Microbiology 12:3150-3158.

Thaiss, C. A., N. Zmora, M. Levy, And E. Elinav. 2016. The microbiome and innate immunity. Nature 535:65-74.

WARnE, R. W., L. Kirschman, AND L. ZeGLin. 2017. Manipulation of gut microbiota reveals shifting community structure shaped by host developmental windows in amphibian larvae. Integrative and Comparative Biology 57:786-794.

WERNER, E. E., AND K. S. GLENNEMEIER. 1999. Influence of forest canopy cover on the breeding pond distributions of several amphibian species. Copeia 1999:1-12.

Woodhams, D. C., H. Brandt, S. Baumgartner, J. Kielgast, E. Küpfer, U. Tobler, L. R. Davis, B. R. Schidid, C. Bel, S. Hodel, et Al. 2014. Interacting symbionts and immunity in the amphibian skin mucosome predict disease risk and probiotic effectiveness. PLoS One 9:e96375.

Wuerthner, V. P., J. Hua, And J. T. Hoverman. 2017. The benefits of coinfection: trematodes alter disease outcomes associated with virus infection. Journal of Animal Ecology 86: 921-931.

Accepted: 15 April 2019

Published online: 5 December 2019 
TABLE A1. List of current experimental amphibian microbiota studies (as of 30 July 2018) showing manuscript citation, host species identity, stressor tested, captive habitat conditions, age of individuals, experiment duration, host source, and microbiota source.

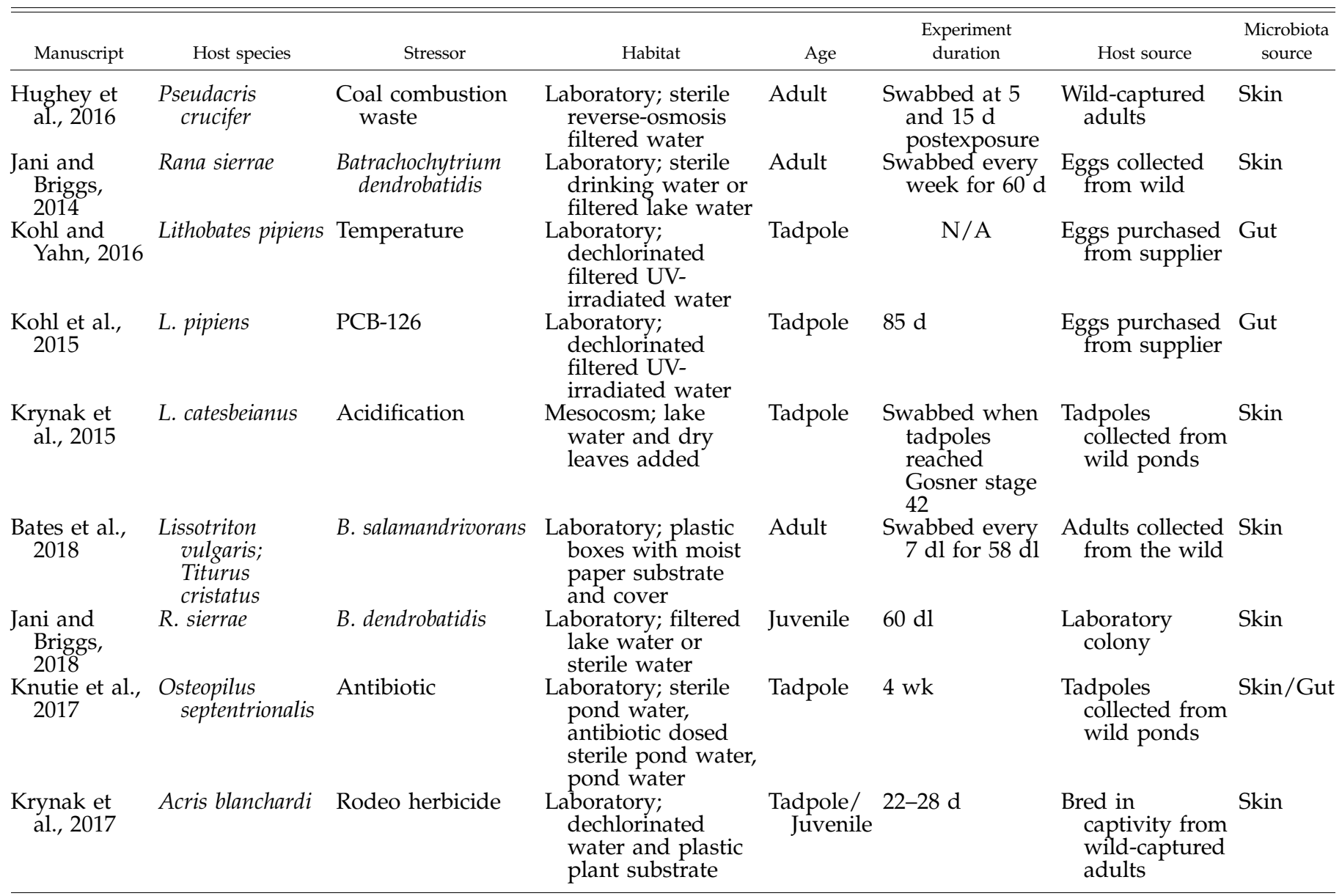

\section{Appendix 1 Literature Cited}

Bates, K. A., F. C. Clare, S. O’Hanlon, J. Bosch, L. Brookes, K. Hopkins, E. J. McLaughlin, O. Daniel, T. W. J. Garner, M. C. Fisher, et Al. 2018. Amphibian chytridiomycosis outbreak dynamics are linked with host skin bacterial community structure. Nature Communications 9: 693.

Hughey, M. C., J. B. Walke, M. H. Becker, T. P. Umile, E. A. Burzynski, K. P. C. Minbiole, A. A. Iannetta, C. N. Santiago, W. A. Hopkins, and L. K. BELDEN. 2016. Short-term exposure to coal combustion waste has little impact on the skin microbiome of adult spring peepers (Pseudacris crucifer). Applied and Environmental Microbiology 82: 3493-3502.

JANI, A. J., AND C. J. BRIGgS. 2014. The pathogen Batrachochytrium dendrobatidis disturbs the frog skin microbiome during a natural epidemic and experimental infection. Proceedings of the National Academy of Sciences 111:E5049-E5058.

. 2018. Host and aquatic environment shape the amphibian skin microbiome but effects on downstream resistance to the pathogen
Batrachochytrium dendrobatidis are variable. Frontiers in Microbiology 9:487.

KNUTIE, S. A., C. L. WiLKInson, K. D. KoHL, AND J. R. Rohr. 2017. Early-life disruption of amphibian microbiota decreases later-life resistance to parasites. Nature Communications 8:86.

Kohl, K. D., T. L. Cary, W. H. Karasov, and M. D. Dearing. 2015. Larval exposure to polychlorinated biphenyl 126 (PCB-126) causes persistent alteration of the amphibian gut microbiota. Environmental Toxicology and Chemistry 34:1113-1118.

KOHL, K. D., AND J. YAHN. 2016. Effects of environmental temperature on the gut microbial communities of tadpoles. Environmental Microbiology 18:1561-1565.

KRYNAK, K. L., D. J. BuRKE, AND M. F. BenARD. 2015. Larval environment alters amphibian immune defenses differentially across life stages and populations. PLoS One 10:e0130383.

- 2017. Rodeo ${ }^{\mathrm{TM}}$ herbicide negatively affects Blanchard's cricket frogs (Acris blanchardi) survival and alters the skin-associated bacterial community. Journal of Herpetology 51:402-410. 
APPENDIX 2
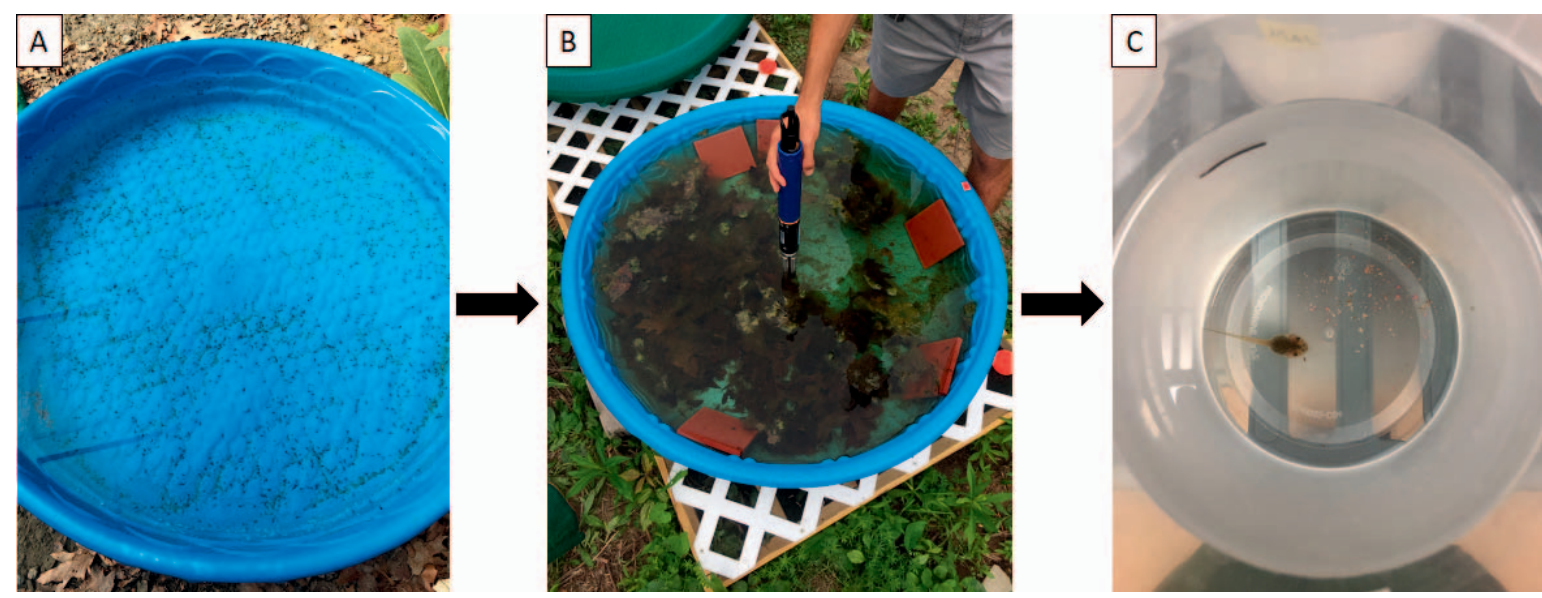

FIG. A1. Photos of the housing conditions utilized in this study. Individuals were moved from (A) common garden culture pools to (B) seminatural mesocosms and finally into (C) laboratory conditions (Table 1). 


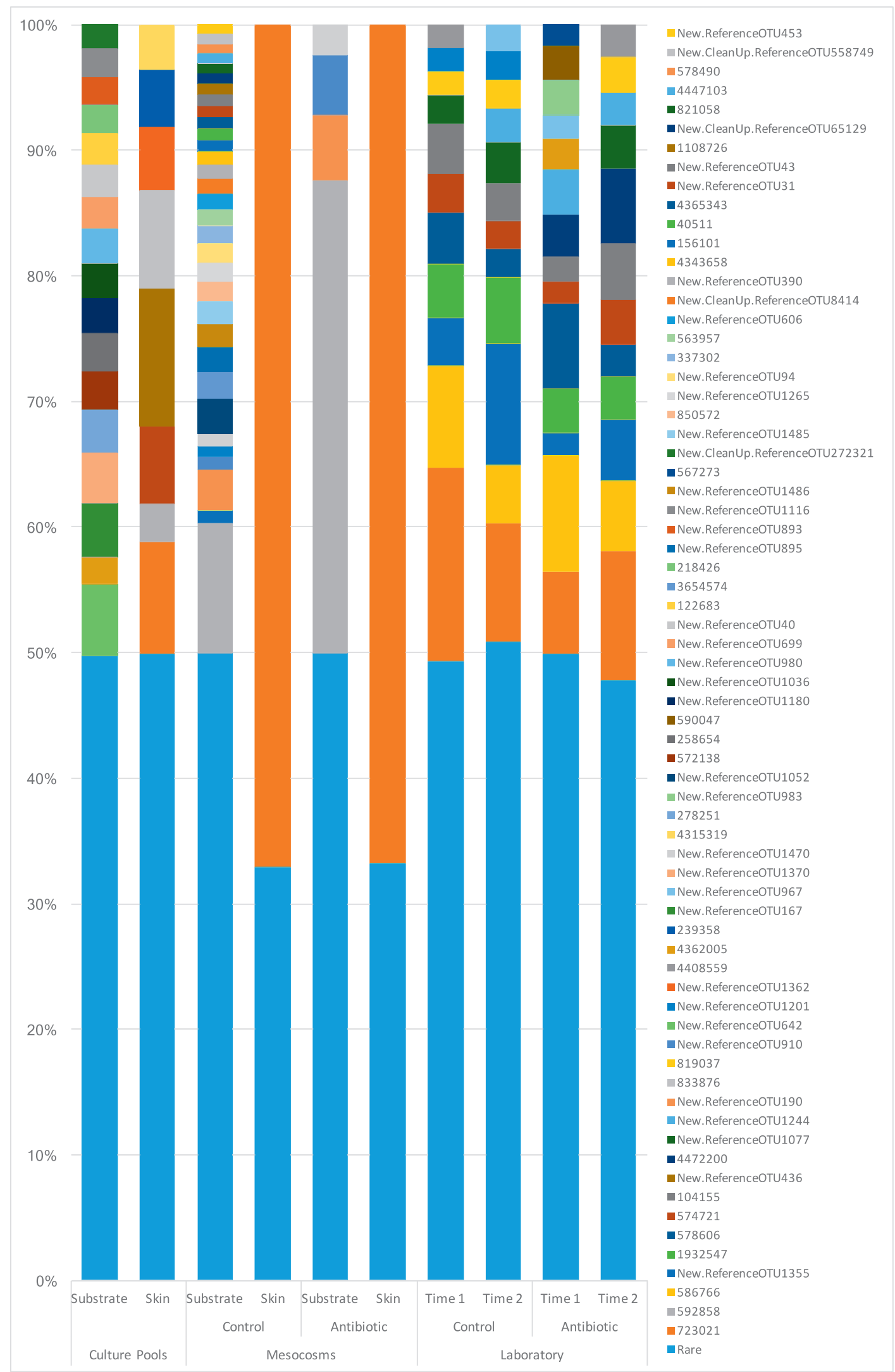

FIG. A2. Relative abundance of most common OTUs (relative abundance $>1.0 \%$ ) characterized on the skin of Northern Leopard Frogs inhabiting three different captive environments. 


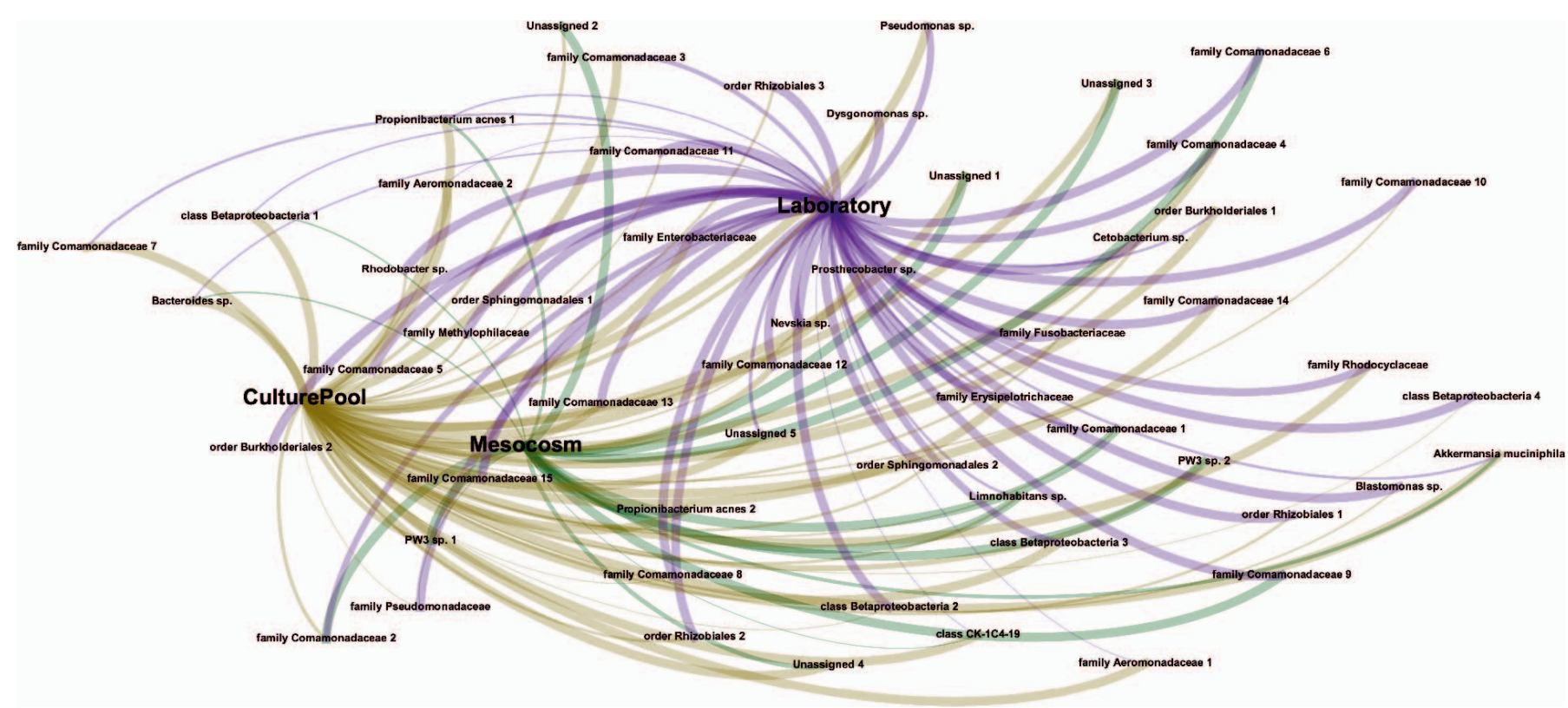

FIG. A3. Bipartite network displaying the distribution of OTUs across the core microbiotas of Northern Leopard Frogs in three rearing environments (core microbiota corresponds to OTUs present in $80 \%$ of individuals). Lines connect OTUs to rearing environment and are weighted by relative abundance.

\section{APPENDIX 3}

Statistical Analyses: Influence of Husbandry Environment on Microbial Community Factors.-To evaluate the effect of husbandry environment on microbial community factors, we tested whether the proportion of shared OTUs between tadpoles and substrate, alpha diversity, and beta diversity varied based on husbandry venue. We implemented a linear mixed model (LMM) using shared OTUs as a dependent variable, venue (culture pool or mesocosm) as a fixed variable, and unit (container) as a random variable using a Gaussian distribution. Next, we assessed whether husbandry venue had an influence on alpha diversity (observed OTUs, Shannon index, and Faith's phylogenetic diversity) using LMMs. We performed three separate LMMs each using alpha metric as a dependent variable, husbandry venue (culture pool, mesocosm, or laboratory) as a fixed factor, and unit as a random factor. We implemented a Gaussian distribution for Shannon index and Faith's phylogenetic index comparisons and a negative binomial distribution for the OTU richness comparisons. These same distributions are used in all subsequent analyses on alpha metrics. In addition, we used Adonis tests to evaluate if husbandry venue explained variation in each beta diversity metric (unweighted UniFrac, weighted UniFrac, and Bray-Curtis dissimilarities), with husbandry venue as a grouping factor.

Influence of SDM Treatment across Space.-We executed analyses testing the influence of husbandry venue (mesocosm and laboratory venues) on intertreatment group alpha and beta diversity variation. We implemented LMMs where we individually tested the three alpha diversity metrics as a dependent variable and SDM treatment as a fixed variable. We included unit ID nested within husbandry venue to account for interunit and interhusbandry venue effects. We then calculated unweighted UniFrac, weighted UniFrac, and BrayCurtis dissimilarity matrices for all laboratory and mesocosm individuals. For each beta diversity metric we calculated the average difference between each SDM-treated individual and the corresponding control samples within each unit. We used average beta diversity differences between SDM-treated and control individuals as a dependent variable in LMMs, husbandry venue as a fixed variable, and unit as a random variable to assess whether husbandry venue influenced the effect of SDM on community composition. To evaluate which OTUs associated with SDMexposure in both environments, we performed an indicator species analysis using the $\mathrm{R}$ package indicspecies. We ran the community data of each environment independently and compared the resulting lists of associations.

Influence of SDM Treatment across Time.-To evaluate the effect of time on the skin microbiome and experiment results, we tested whether time in the laboratory had an influence on alpha and beta diversity variation between control and treatment individuals. To assess the influence of time in captivity on alpha diversity, we used three separate LMMs for each alpha metric, with alpha diversity as a dependent variable, time point, treatment status, and their interaction as fixed factors, and individual identity as a random factor to account for repeated measures. We implemented two-way Adonis tests to evaluate whether treatment and time correlated with grouping of samples. Next, as before, we calculated the average differences between each treatment individual and control samples within each time point and used these values as a dependent variable in an LMM with time point as a fixed factor and individual identity as a random factor. 